\title{
Erratum to: Expression of $h s r \omega-R N A i$ transgene prior to heat shock specifically compromises accumulation of heat shock-induced Hsp70 in Drosophila melanogaster
}

\author{
Anand K. Singh ${ }^{1} \cdot$ Subhash C. Lakhotia $^{1}$
}

Published online: 20 October 2015

(C) Cell Stress Society International 2015

\section{Erratum to: Cell Stress and Chaperones}

DOI 10.1007/s12192-015-0644-6

Due to an unfortunate turn of events, two identifiers in the caption of Fig. 7 a were transposed causing an error in the last sentence of the caption. The correct caption of Fig. $7 \mathrm{a}$ is published here and should be treated as definitive by the reader.
Fig. $7 \mathrm{Hsc} 70$ levels are not increased in hsrw-RNAi transgeneexpressing cells, but post-translational stability of the heat shockinduced Hsp70 is compromised. a Western blot showing sequential detection of $\mathrm{Hsc} 70 / \mathrm{Hsp} 70$ and $\mathrm{Hsp} 70$ using 7.10.3 and $7 \mathrm{Fb}$ antibodies, respectively, in control and heat-shocked WT (B) and Act$G A L 4>h s r \omega-R N A i$ (A) larvae.

The online version of the original article can be found at http://dx.doi.org/ 10.1007/s12192-015-0644-6.

Subhash C. Lakhotia

lakhotia@bhu.ac.in

1 Cytogenetics Laboratory, Department of Zoology, Banaras Hindu University, Varanasi 221005, India 